\title{
Влияние электродов на параметры солнечно-слепых детекторов УФ излучения
}

\author{
(C) В.М. Калыгина, А.В. Цымбалов, А.В. Алмаев, Ю.С. Петрова
}

Национальный исследовательский Томский государственный университет, 634050 Томск Россия

E-mail: Kalygina@ngs.ru

Поступила в Редакцию 28 октября 2020 г.

В окончательной редакции 3 ноября 2020 г.

Принята к публикации 3 ноября 2020 г.

\begin{abstract}
Исследовано влияние топологии электродов на электрические и фотоэлектрические характеристики структур металл/полупроводник/металл. Пленки оксида галлия получали высокочастотным магнетронным распылением мишени $\mathrm{Ga}_{2} \mathrm{O}_{3}$ на сапфировые подложки с ориентацией (0001). На поверхности оксидных пленок были сформированы два типа электродов: два параллельных электрода, с межэлектродным расстоянием 250 мкм и встречно-штырьевые. В электродах второго типа расстояние между „пальцами“ составляло 50, 30, 10 и 5 мкм. Независимо от типа контактов структуры обнаруживают чувствительность к ультрафиолетовому излучению с длиной волны $\lambda=254$ нм. Детекторы второго типа с межэлектродным расстоянием 5 мкм демонстрируют наибольшие значения фототока $I_{\mathrm{ph}}=3.8 \mathrm{MA}$ и удельную обнаружительную способность $D^{*}=5.54 \cdot 10^{15} \mathrm{cM} \cdot \Gamma_{ц}^{0.5} \cdot \mathrm{BT}^{-1}$.
\end{abstract}

Ключевые слова: оксид галлия, RFMS, детекторы, ультрафиолет, электроды.

DOI: 10.21883/FTP.2021.03.50605.9545

\section{1. Введение}

Оксид галлия $\left(\mathrm{Ga}_{2} \mathrm{O}_{3}\right)$ является бинарным полупроводниковым соединением, которое может кристаллизоваться в пяти модификациях: $\alpha-, \beta-, \gamma$-, $\delta$ - и $\varepsilon$ [1-3]. Благодаря своим физико-химическим свойствам этот широкозонный полупроводник представляет практический интерес для создания солнечно-слепых детекторов ультрафиолетового (УФ) диапазона, высоковольтных приборов, газовых сенсоров, беспроводной коммуникации и т.д. $[4,5]$. На сегодняшний день наиболее перспективным для производства УФ детекторов является $\beta-\mathrm{Ga}_{2} \mathrm{O}_{3}$, имеющий ширину запрещенной зоны $E_{g} \sim 4.8$ эВ. Эта модификация оксида галлия обладает высокой химической стабильностью вплоть до температуры плавления $\left(1780^{\circ} \mathrm{C}\right)[6-8]$.

Существует множество методов получения пленок оксида галлия: молекулярно-лучевая эпитаксия (MBE), осаждение металлорганических соединений из газовой фазы (MOCVD), хлорид-гидридная газофазная эпитаксия (HVPE), атомно-слоевое осаждение (ALD), импульсное лазерное напыление (PLD), осаждение металлорганических соединений из газовой фазы при пониженном давлении (LPCVD), высокочастотное магнетронное распыление (RMFS) [9]. Среди перечисленных методов выделяется высокочастотное магнетронное распыление. Данная методика отличается низкой стоимостью, высокой скоростью и хорошей адгезией на больших площадях осаждения, простотой нанесения, гибкостью выбора подложки и мишени [10]. Показано, что пленки оксида галлия, нанесенные с помощью RMFS при температуре $<400^{\circ} \mathrm{C}$, находятся в аморфном состоянии [8]. Отжиг полученных пленок при температуре $900^{\circ} \mathrm{C}$ и выше приводит к формированию поликристаллической $\beta-\mathrm{Ga}_{2} \mathrm{O}_{3}[11,12]$.

В табл. 1 для сравнения приведены характеристики детекторов, в которых оксидную пленку формировали несколькими методами, включая ВЧ-магнетронное напыление: $R$ - значение отклика; $I_{D}-$ темновой ток; $\lambda$ - длина волны падающего излучения; $I_{L} / I_{D}-$ отношение тока, полученного при освещении детектора излучением, к темновому току; Bias - приложенное напряжение.

Из данных табл. 1 можно сделать вывод о том, что RFMS метод позволяет получать детекторы с высокими фотоэлектрическими характеристиками, которые сопоставимы с параметрами детекторов, полученных при использовании других более дорогих и сложных методов изготовления.

\section{2. Методика эксперимента}

В работе были получены два типа детекторов: детекторы с двумя параллельными электродами (далее первый тип образцов) и детекторы со встречно-штырьевыми электродами (далее второй тип образцов).

В детекторах первого типа электроды создавали путем нанесения слоя платины на гладкие сапфировые подложки методом вакуумного напыления с последующим формированием контактов при помощи взрывной фотолитографии. Расстояние между электродами составляло 250 мкм (рис. 1,a). Пленку оксида галлия толщиной 150-200 нм наносили ВЧ-магнетронным распылением мишени $\mathrm{Ga}_{2} \mathrm{O}_{3}(99.999 \%)$ на не нагретые сапфировые подложки на установке AUTO-500 (производитель Edwards) в газовой смеси $\mathrm{Ar} / \mathrm{O}_{2}$. Концентрация кислоро- 
Таблица 1. Сравнение основных характеристик фотоприемников MSM на основе $\mathrm{Ga}_{2} \mathrm{O}_{3}$

\begin{tabular}{|c|c|c|c|c|c|c|c|}
\hline Метод & Структура & $R, \mathrm{~A} / \mathrm{BT}_{\mathrm{T}}$ & $I_{D}, \Pi \mathrm{A}$ & $\lambda$, нм & Соотношение, $I_{L} / I_{D}$ & Смещение, В & Ссылка \\
\hline RMFS & MSM $\mathrm{Ga}_{2} \mathrm{O}_{3}$ на кварце & 436.3 & 4.9 & 240 & $10.7 \cdot 10^{4}$ & 25 & [13] \\
\hline RMFS & MSM $\beta-\mathrm{Ga}_{2} \mathrm{O}_{3}$ на сапфире & - & 100 & 254 & $16.25 \cdot 10^{3}$ & 5 & {$[9]$} \\
\hline RMFS & MSM Ga ${ }_{2} \mathrm{O}_{3}$ на кварце & 91.88 & 200 & 254 & $>10^{4}$ & 10 & [14] \\
\hline RMFS & $\mathrm{MSM} \mathrm{Ga} / \mathrm{Ga}_{2} \mathrm{O}_{3}$ & 2.85 & 8.52 & 260 & $8 \cdot 10^{5}$ & 15 & {$[15]$} \\
\hline RMFS & MSM Au плазмон $\beta-\mathrm{Ga}_{2} \mathrm{O}_{3}$ на $\mathrm{Si}$ & 50 & 280 & 254 & - & - & [16] \\
\hline MOCVD & MSM $\beta-\mathrm{Ga}_{2} \mathrm{O}_{3}$ на сапфире & 17 & 1850 & 255 & $8.5 \cdot 10^{6}$ & 20 & {$[17]$} \\
\hline MBE & MSM $\beta-\mathrm{Ga}_{2} \mathrm{O}_{3}$ на сапфире & $3.7 \cdot 10^{-2}$ & 1.2 & 254 & - & 10 & [18] \\
\hline PLD & MSM $\beta-\mathrm{Ga}_{2} \mathrm{O}_{3}$ на сапфире & 0.9 & - & 254 & $1.8 \cdot 10^{5}$ & 5 & {$[19]$} \\
\hline LPCVD & MSM $\beta-\mathrm{Ga}_{2} \mathrm{O}_{3}$ на сапфире & 0.14 & - & 250 & $1.44 \cdot 10^{6}$ & 1 & [20] \\
\hline
\end{tabular}

да в смеси поддерживалась равной $(56.1 \pm 0.5)$ об\%. Расстояние между мишенью и подложкой составляло 70 мм. Давление в камере во время напыления поддерживалось равным $7 \cdot 10^{-6}$ бар. На следующем этапе сапфировую пластину с нанесенными Pt электродами и оксидной пленкой подвергали отжигу в аргоне при $900^{\circ} \mathrm{C}$ в течение 30 мин. Затем пластину разрезали на чипы размером $1.4 \times 1.4$ мм. Изготовление детекторов заканчивалось распайкой Pt-контактов на кристалл-держатель с помощью золотой проволоки диаметром 50 мкм и размещением образцов в металлические корпуса ТО-8.

Второй тип образцов был изготовлен аналогичным образом, за исключением того, что сначала на гладкую сапфировую подложку ВЧ-магнетронным распылением мишени $\mathrm{Ga}_{2} \mathrm{O}_{3}$ напылялась пленка оксида галлия, a затем формировалась встречно-штырьевая топология электродов на основе Ti/V. В результате были получены 4 вида образов с межэлектродным расстоянием $d$, равным 50, 30, 10 и 5 мкм. Количество электродов составляло 50, 75, 150 и 200 соответственно. Независимо от межэлектродного расстояния размер чипов равнялся $3.5 \times 3.5$ мм (рис. $1, b$ ).

Фазовый состав пленок оксида галлия исследовали при комнатной температуре на установке Renishaw inVia
Basis (производство Германия). Пространственное разрешение системы составляло 1 мкм. Образцы облучались твердотельным лазером Renishaw с длиной волны 532 нм и выходной мощностью излучения $100 \mathrm{MBT}$.

Анализ элементного состава образцов осуществлялся с помощью оже-спектроскопии на установке „Шхуна-2“. Разрешение оже-анализатора по энергии составляло $0.1 \%$. В анализатор коаксиально встроен источник электронов, формирующий зондирующий пучок диаметром 1 мкм. Энергия электронов в пучке равнялась 3 кэВ. Для распыления поверхности исследуемого образца использовался пучок ионов аргона, диаметр которого можно изменять от 0.5 до 2 мм. Пучок ионов формировался источником, ось которого наклонена на $70^{\circ}$ относительно нормали к поверхности исследуемого образца. Энергия ионов $\mathrm{Ar}$ составляла 3 кэВ.

Темновые вольт-амперные характеристики (BAX) и BAX при воздействии ультрафиолетового излучения исследовали при комнатной температуре с помощью источника-измерителя Keithley 2611B. В качестве источника УФ излучения использовалась криптон-фторовая лампа VL-6.C с фильтром на 254 нм. Расстояние между лампой и образцами составляло $1 \mathrm{~cm}$, а падающая интенсивность излучения равнялась $1.65 \mathrm{MBT} / \mathrm{cm}^{2}$. a

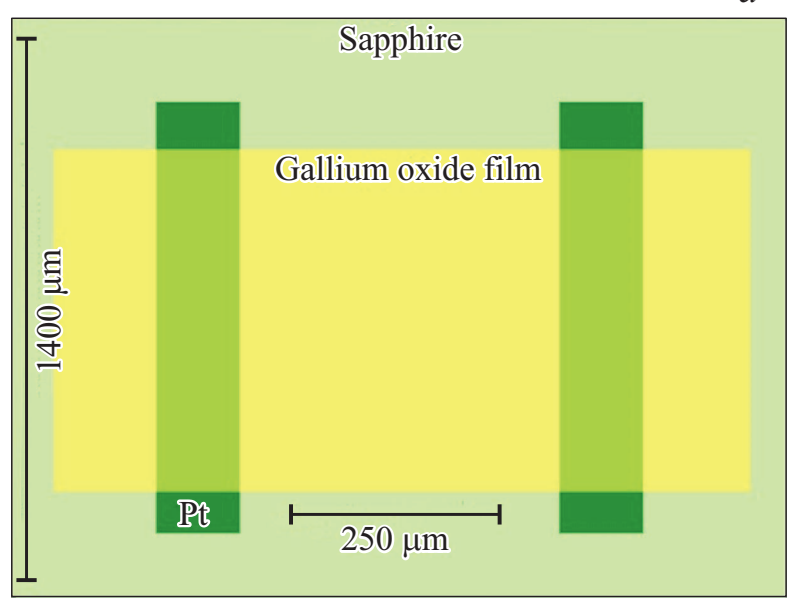

$b$

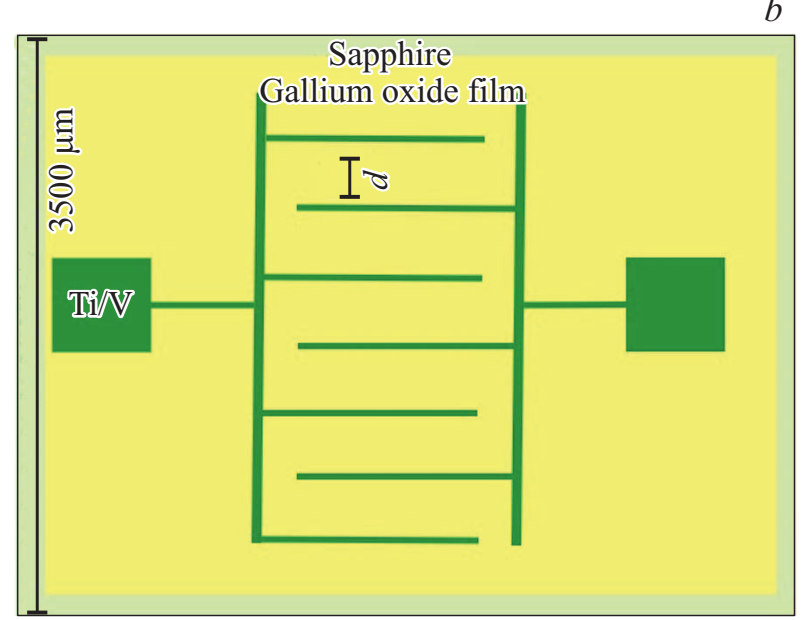

Рис. 1. Схематическое изображение активных областей детекторов первого $(a)$ и второго видов $(b)$. 


\section{3. Результаты эксперимента и их обсуждение}

На рис. 2 представлен рамановский спектр отожженных пленок оксида галлия. Пики при 418, 577 и $750 \mathrm{~cm}^{-1}$ соответствуют сапфировой подложке [21,22]. Оставшиеся шесть мод принадлежат к $\beta-\mathrm{Ga}_{2} \mathrm{O}_{3}$. Их можно разделить на 3 группы: моды, обусловленные высокочастотным растяжением и изгибом тетраэдров $\mathrm{GaO}_{4}$ (770-500 $\left.\mathrm{cm}^{-1}\right)$; среднечастотные моды, обусловленные деформацией октаэдров $\mathrm{Ga}_{2} \mathrm{O}_{6}\left(480-500 \mathrm{~cm}^{-1}\right)$; низкочастотные моды, связанные с либрацией и трансляцией цепочек, состоящих из тетраэдров и октаэдров $\left(<200 \mathrm{~cm}^{-1}\right)$. Из полученных результатов следует, что высокотемпературный отжиг приводит к формированию поликристаллической пленки $\beta$-фазы оксида галлия $[23,24]$.

При помощи оже-спектроскопии была получена зависимость элементного состава активной области детекто-

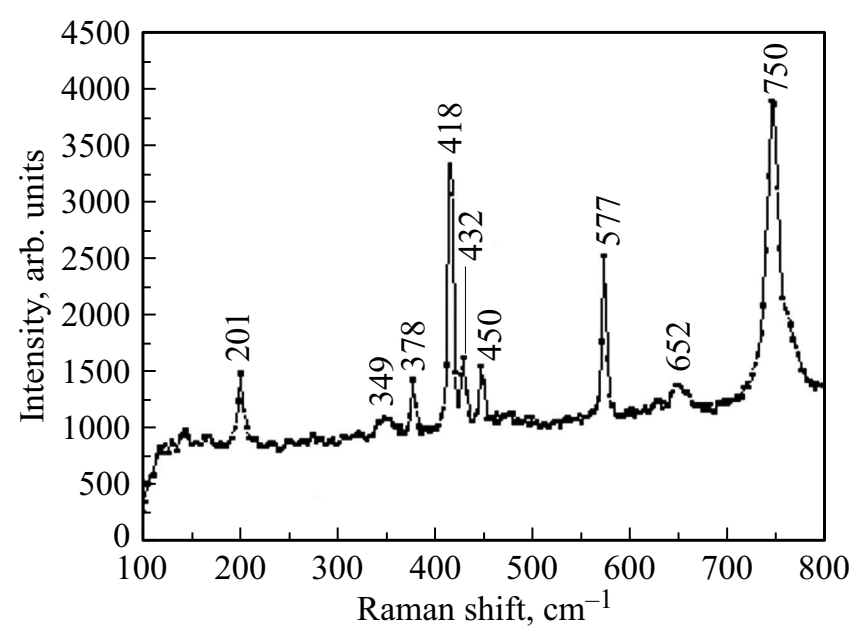

Рис. 2. Рамановский спектр отожженных пленок оксида галлия.

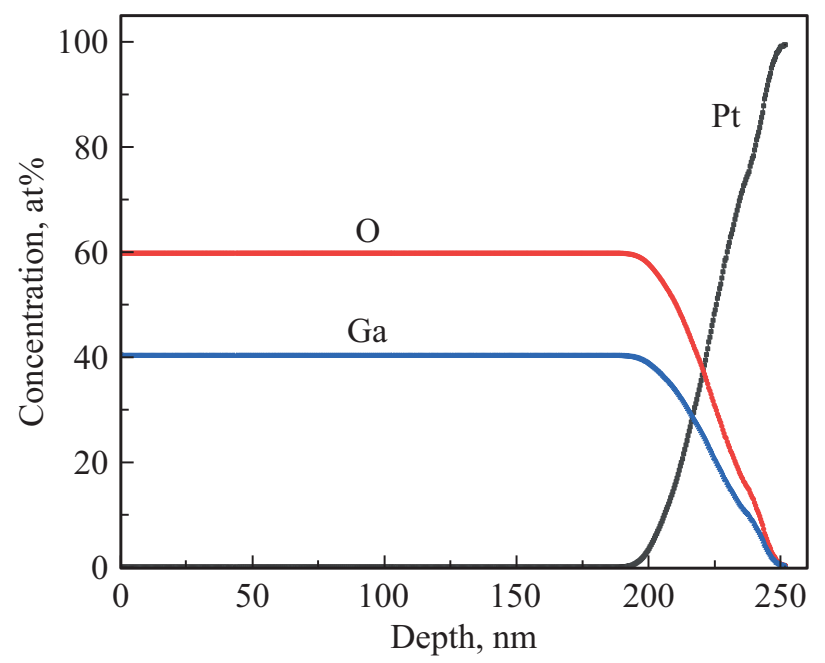

Рис. 3. Зависимость элементного состава активной области детекторов первого типа от ее толщины. ров первого типа от ее толщины (рис. 3). Приведенные данные показывают, что пленка оксида галлия обладает высокой стехиометрией, которая близка к 60/40 ат\% $(\mathrm{O} / \mathrm{Ga})$. Начиная с толщины $\sim 190 \mathrm{Hм}$ стехиометрия $\mathrm{Ga}_{2} \mathrm{O}_{3}$ начинает монотонно ухудшаться, что обусловлено наличием платиновых контактов под пленкой.

Для образцов обоих типов вольт-амперные (BAX) характеристики симметричны относительно полярности напряжения. Зависимость темнового тока $\left(I_{D}\right)$ от напряжения $(U)$ в детекторах первого типа имеет линейный вид. Значения $I_{D}$ не превышают $0.2-35$ нА в интервале приложенных напряжений от $-200 \leq 0 \leq 200$ В. Вольтамперная характеристика, полученная при освещении образца светом с длиной волны $\lambda=254$ нм, сохраняет линейную зависимость. Ультрафиолетовое излучение приводит к росту тока в активной области детектора на 2-3 порядка. Значения тока при освещении $\left(I_{L}\right)$ находятся в диапазоне 4-540 нА при напряжениях от 0 до $\pm 200 \mathrm{~B}$.

Детекторы второго типа в отсутствие УФ излучения демонстрируют квазилинейный вид $\mathrm{BAX}$ (рис. 4, $a$ ). Воздействие излучения с длиной волны $\lambda=254$ нм приводит к увеличению тока на 5-7 порядков (в зависимости от образца) и изменению характера зависимости тока от напряжения. Для всех образцов второго типа ВАХ принимает вид кривой, имеющей участок насыщения $I_{L}$ (рис. $4, b)$. С уменьшением межэлектродного расстояния снижается напряжение $U_{m}$, соответствующее началу участка насыщения на кривых зависимостей $I_{L}$ от напряжения (табл. 2).

Следует заметить, что насыщение на световых ВАХ наблюдается, когда $I_{L}$ приближается или достигает единиц миллиампер. В табл. 2 приведены значения $I_{L}\left(U_{m}\right)$ при напряжениях, соответствующих началу участка насыщения, и даны значения мощности $P_{m}$, выделяемой в образцах при указанных условиях. С учетом этих оценок можно предположить, что на форму BAX при больших значениях $I_{L}$, по-видимому, влияет нагрев образцов. Мощность, при которой наблюдается участок насыщения, незначительно отличается для детекторов с различным межэлектродным расстоянием.

Увеличение фототока в детекторах второго типа, по сравнению с детекторами первого типа, связано с повышением коэффициента ударной ионизации, который возрастает с напряженностью электрического поля [25].

Для расчета отклика $R$, удельной обнаружительной способности $D^{*}$ и внешней квантовой эффективности $\eta$ детекторов использовались следующие выражения [19]:

$$
\begin{aligned}
R & =\frac{I_{L}-I_{D}}{P}, \\
D^{*} & =R \sqrt{\frac{S}{2 e I_{D}}}, \\
\eta & =\frac{h c R}{e \lambda}
\end{aligned}
$$

где $I_{L}-I_{D}=I_{\mathrm{ph}}-$ фототок, $P-$ падающая мощность излучения на активную область детектора, $S$ - площадь 
Таблица 2. Зависимость от межэлектродного расстояния: темнового тока, фототока, тока во время действия УФ в начале участка насыщения, напряжения насыщения и мощности в начале участка насыщения

\begin{tabular}{c|c|c|c|c|c}
\hline$d$, мкм & $I_{D}, \mathrm{~A}(U=10 \mathrm{~B})$ & $I_{L}, \mathrm{~A}(U=10 \mathrm{~B})$ & $I_{L}\left(U_{m}\right), \mathrm{A}$ & $U_{m}, \mathrm{~B}$ & $P_{m}, \mathrm{BT}$ \\
\hline 250 & $3.3 \cdot 10^{-11}$ & $2.65 \cdot 10^{-8}$ & - & - & - \\
50 & $1 \cdot 10^{-10}$ & $1.52 \cdot 10^{-4}$ & $5.5 \cdot 10^{-4}$ & 165 & 0.09 \\
30 & $6.7 \cdot 10^{-11}$ & $1.44 \cdot 10^{-4}$ & $6.2 \cdot 10^{-4}$ & 160 & 0.10 \\
10 & $6 \cdot 10^{-11}$ & $8 \cdot 10^{-4}$ & $2.7 \cdot 10^{-3}$ & 40 & 0.11 \\
5 & $4.3 \cdot 10^{-11}$ & $3.8 \cdot 10^{-3}$ & $6.7 \cdot 10^{-3}$ & 19 & 0.13
\end{tabular}
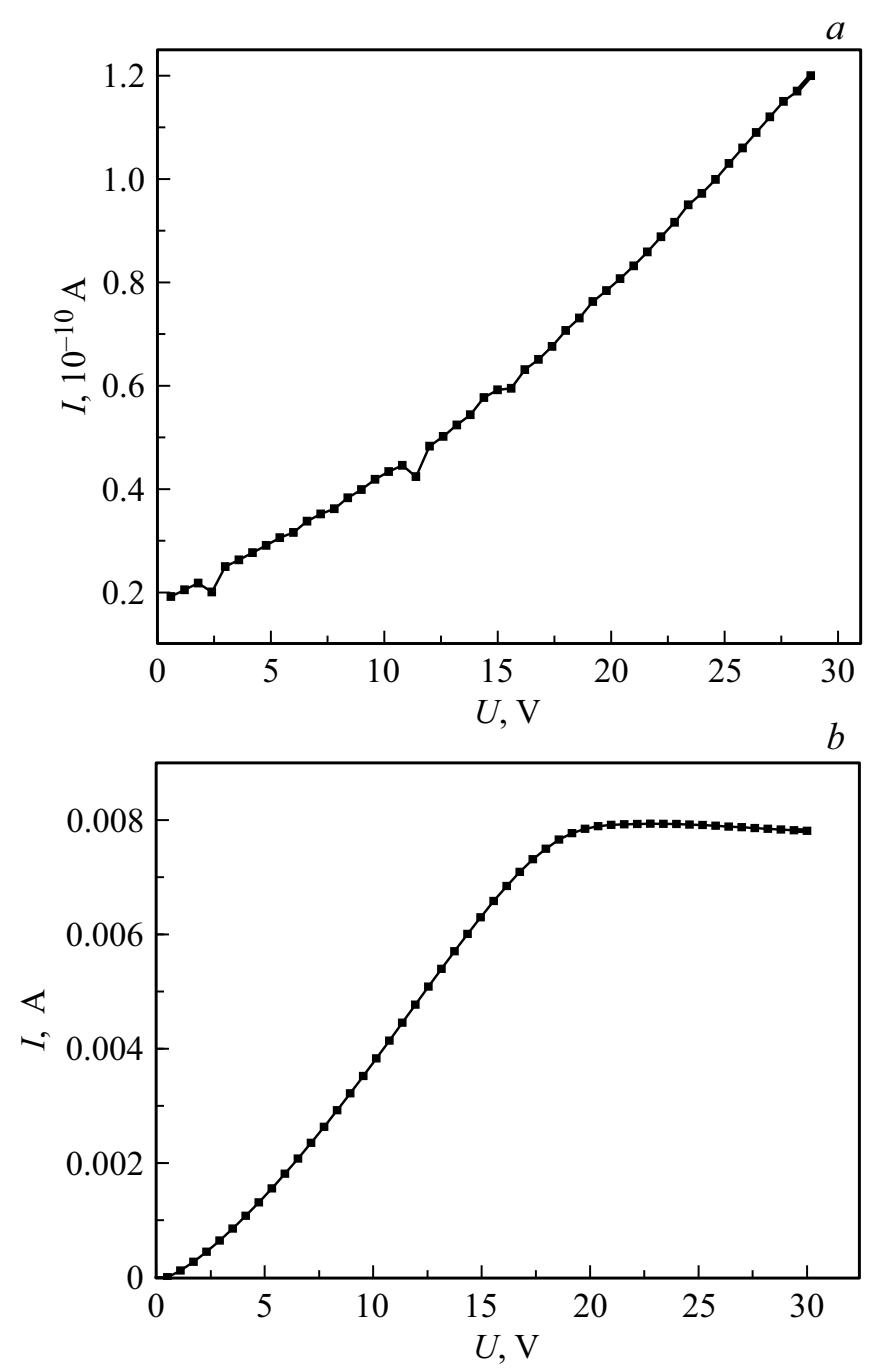

Рис. 4. ВАХ детектора второго типа с межэлектродным расстоянием 5 мкм, измеренная в отсутствие $(a)$ и под действием излучения длиной волны $\lambda=254$ нм $(b)$.

активной области, $e$ - заряд электрона, $h$ - постоянная Планка, $c$ - скорость света, $\lambda$ - длина волны падающего излучения.

В табл. 3 представлены фотоэлектрические характеристики для всех типов детекторов при фиксированном напряжении $10 \mathrm{~B}$, рассчитанные при использовании соотношений (1)-(3). Из данных следует, что уменьшение
Таблица 3. Фотоэлектрические характеристики детекторов при фиксированном напряжении $10 \mathrm{~B}$

\begin{tabular}{r|c|c|c|c}
\hline$d$, мкм & $\begin{array}{c}D^{*}, \\
\mathrm{cм} \cdot \Gamma^{0.5} \cdot \mathrm{BT}^{-1}\end{array}$ & $\eta$ & $R, \mathrm{~A} / \mathrm{BT}$ & $\begin{array}{c}\text { Соотношение, } \\
I_{L} / I_{D}\end{array}$ \\
\hline 250 & $4 \cdot 10^{11}$ & 0.05 & 0.01 & 800 \\
50 & $1 \cdot 10^{14}$ & 16.7 & 3.4 & $1.5 \cdot 10^{6}$ \\
30 & $2 \cdot 10^{14}$ & 17.3 & 3.5 & $2.15 \cdot 10^{6}$ \\
10 & $8.4 \cdot 10^{14}$ & 135 & 27.8 & $1.3 \cdot 10^{7}$ \\
5 & $5.54 \cdot 10^{15}$ & 896 & 183 & $1 \cdot 10^{8}$
\end{tabular}

межэлектродного расстояния ведет к увеличению всех параметров детекторов. Влияние топологии электродов можно заметить, если сравнивать характеристики детекторов первого и второго типов.

\section{4. Заключение}

Полиморфные пленки оксида галлия после отжига обнаруживают высокую стехиометрию и содержат исключительно $\beta-\mathrm{Ga}_{2} \mathrm{O}_{3}$. Детекторы со встречно-штырьевыми электродами имеют фотоэлектрические характеристики на несколько порядков лучше, чем детекторы с двумя параллельными сплошными электродами.

Темновые токи детекторов слабо зависят от конструкции контактов. Детекторы со встречно-штыревыми электродами имеют ВАX с участком насыщения, который смещается в область низких напряжений с уменьшением межэлектродного расстояния. Лучшие значения фотоэлектрических характеристик имеют детекторы второго типа с межэлектродным расстоянием 5 мкм.

\section{Финансирование}

Работа выполнена при поддержке гранта Российского научного фонда № 20-79-10043.

\section{Конфоликт интересов}

Авторы заявляют, что у них нет конфликта интересов. 


\section{Список литературы}

[1] S. Lee, Y. Ito, K. Kaneko, S. Fujita. Jpn. J. Appl. Phys., 54, 3 (2015).

[2] A.A. Dakhel. Sol. St. Sci., 20, 54 (2013).

[3] S.I. Stepanov, V.I. Nikolaev, V.E. Bougrov, A.E. Romanov. Rev. Adv. Mater. Sci., 44, 63 (2016).

[4] I. Cora, F. Mezzadri, F. Boschi, M. Bosi, M. Caplovicova, G. Calestani, I. Dodony, B. Pecza, R. Fornari. Cryst. Eng. Commun., 19, 1509 (2017).

[5] K. Li, Xun Yang, Y. Tian, Y. Chen, C. Lin, Z. Zhang, Z. Xu, J. Zang, C. Shan. Sci. China Phys., Mech., 63, 117312 (2020).

[6] D. Guo, X. Qin, M. Lv, H. Shi, Y. Su, G. Yao, S. Wang, C. Li, P. Li, W. Tang. Electron. Mater. Lett., 13, 483 (2017).

[7] L. Qian, Z. Wu, Y. Zhang, P. Lai, X. Liu, Y. Li. ACS Photonics, 4, 2203 (2017).

[8] J.W. Roberts, P.R. Chalker, B. Ding, R.A. Oliver, J.T. Gibbon, L.A.H. Jones, V.R. Dhanak, L.J. Phillips, L.J. Major, F.C. Massabuau. J. Cryst. Growth, 528, 125254 (2019).

[9] X. Wang, Z. Chen, D. Guo, X. Zhang, Z. Wu, P. Li, W. Tang. Optical Mater. Express, 8, 2918 (2018).

[10] A.K. Saikumar, S. Nehate, K.B. Sundaram. ECS J. Solid State Sci. Technol., 8, 3064 (2019).

[11] P. Schurig, F. Michel, A. Beyer, K. Volz, M. Becker, A. Polity, P. Klar. Phys. Status Solidi A, 217, 1901009 (2020).

[12] V.M. Kalygina, T.Z. Lygdenova, V.A. Novikov, Yu.S. Petrova, A.V. Tsymbalov, T.M. Yaskevich. Semiconductors, 53, 388 (2019).

[13] S. Han, X. Huang, M. Fang, W. Zhao, S. Xu, D. Zhu, W. Xu, M. Fang, W. Liu, P. Caoa, Y. Lua. J. Mater. Chem. C, 7, 11834 (2019).

[14] S. Cui, Z. Mei, Y. Zhang, H. Liang, X. Du. Adv. Opt. Mater., 5, 1700454 (2017).

[15] S. Cui, Z. Mei, Y. Hou, Q. Chen, H. Liang, Y. Zhang, W. Huo, X. Du. Chin. Physics B, 27, 067301 (2018).

[16] Y. An, X. Chu, Y. Huang, Y. Zhi, D. Guo, P. Li, Z. Wu, W. Tang. Progr. Nat. Sci., 26, 65 (2016).

[17] G.C. Hu, C.X. Shan, Nan Zhang, M.M. Jiang, S.P. Wang, D.Z. Shen. Opt. Express, 23, 13554 (2015).

[18] T. Oshima, T. Okuno, S. Fujita. Jpn. J. Appl. Phys., 46, 11 (2007).

[19] F. Yu, S. Ou, D. Wuu. Optical Mater. Express, 5, 1240 (2015).

[20] S. Rafique, L. Han, H. Zhao. Phys. Status Solidi A, 214, 1700063 (2017).

[21] W. Jia, W.M. Yen. J. Raman Spectrosc., 20, 785 (1989).

[22] M.G. Mynbaeva, P.S. Shirshnev, A.V. Kremleva, A.N. Smirnov, E.V. Ivanova, M.V. Zamoryanskaya, I.P. Nikitina, A.A. Lavrent'ev, K.D. Mynbaeva, M.A. Odnoblyudov, D.A. Bauman, H. Lipsanen, V.I. Nikolaev, V.E. Bougrov, A.E. Romanov. Rev. Adv. Mater. Sci., 57, 97 (2019).

[23] C. Kranert, C. Sturm, R. Grund, M. Grundmann. Scientific Rep., 6, 35964 (2016).

[24] M. Kadleikova, J. Breza, M. Vesely. Microelectronics J., 32, 955 (2001).

[25] А.В. Войцеховский, И.И. Ижнин, В.П. Савчин, Н.М. Вакив. Физические основы полупроводниковой фотоэлектроники (Томск, Томский гос. ун-т, 2013).

\section{Influence of electrode topology on the parameters of solar-blind UV detectors}

\section{V.M. Kalygina, A.V. Tsymbalov, A.V. Almaev,} Yu.S. Petrova

\section{National Research Tomsk State University, 634050 Tomsk, Russia}

Abstract The paper investigates the influence of electrode topology on the electrical and photoelectric characteristics of metal/semiconductor/metal structures. Gallium oxide films were obtained by radio-frequency sputtering of a $\mathrm{Ga}_{2} \mathrm{O}_{3}$ target onto sapphire substrates with the (0001) orientation. Two types of electrodes were formed on the surface of the oxide films: two parallel electrodes with an interelectrode distance of $250 \mu \mathrm{m}$ and interdigitated ones. The distance between the "fingers" of the detectors of the second type was $50,30,10$, and $5 \mu \mathrm{m}$. Regardless of the type of contacts, the structures exhibit sensitivity to ultraviolet radiation with a wavelength of $\lambda=254 \mathrm{~nm}$. Detectors of the second type with an interelectrode distance of $5 \mu \mathrm{m}$ demonstrate the highest values of the photocurrent $I_{\mathrm{ph}}=3.8 \mathrm{~mA}$ and detectivity $D^{*}=5.54 \cdot 10^{15} \mathrm{~cm} \cdot \mathrm{Hz}^{0.5} \cdot \mathrm{W}^{-1}$. 